\title{
Optimización estadística del medio de cultivo de una bacteria del género Pseudomonas sp. para la producción de biosurfactantes
}

\section{Statistic optimization of Pseudomonas sp. culture media for biosurfactant production}

Presentación: 6-7/10/2020

\section{Doctorando:}

\section{Franco Andrés Liporace}

Laboratorio de Biotecnología, Facultad Regional Delta, Universidad Tecnológica Nacional - Argentina. fliporace@frd.utn.edu.ar

\section{Director/es:}

\section{Carla Verónica Quevedo}

\section{Resumen}

El objetivo del presente trabajo es obtener la temperatura y concentración de glicerol óptima en el medio de cultivo utilizado para la producción de biosurfactantes a partir de una cepa de Pseudomonas $s p$. La optimización se llevó a cabo a través de una metodología de superficie de respuesta utilizando un diseño central compuesto centrado en las caras, donde concentración de glicerol y temperatura fueron los factores y concentración de biosurfactante la respuesta. Se halló un modelo matemático basado en los resultados experimentales que permite predecir la influencia de los factores en la producción de biosurfactantes. De acuerdo con dicho modelo, una concentración de $7,6 \% \mathrm{v} / \mathrm{v}$ y una temperatura de $25,9^{\circ} \mathrm{C}$ harían máxima la respuesta. En estas condiciones dicha respuesta tendría un valor de $0,43 \mathrm{~g} / \mathrm{l}$.

Palabras clave: Biosurfactantes, Metodología de superficie de respuesta, Pseudomonas sp.

\begin{abstract}
The aim of the present work is to obtain the optimal temperature and the optimal concentration of glycerol in culture media for biosurfactant production using a bacterial strain of the Pseudomonas sp. Optimization was carried out through response surface methodology using a face centered-central composite design, where analytical grade glycerol and temperature were the factors and biosurfactant production the response variable. We found a mathematical model based on experimental results that predicts the influence of the factors on biosurfactant production. According to the model, 7,6\%v/v of glycerol and $25,9^{\circ} \mathrm{C}$ were the optimal values. Here, the predicted biosurfactant concentration would be $0,43 \mathrm{~g} / \mathrm{l}$.
\end{abstract}

Keywords: Biosurfactants, Response surface methodology, Pseudomonas sp.

\section{Introducción}

El auge y la necesidad industrial de los compuestos petroquímicos hace que se produzcan mil millones de toneladas de desechos anuales a nivel mundial (Adams, Fufeyin, Okoro, Ehinomen, \& Biology, 2015). La mala implementación o falta de sistemas de disposición final de residuos provoca que estos contaminen el entorno (Bioremediation_A review of applications and problems to be solved.pdf, n.d.). Más del $90 \%$ de los daños ambientales 
por hidrocarburos $(\mathrm{HC})$ está directamente relacionado con accidentes debidos a fallas humanas, incluida la eliminación deliberada de desechos (Allard \& Neilson, 1997). Tecnologías nuevas, alternativas y mejoradas de recuperación de petróleo se estudian ampliamente en la actualidad (Doheim, 2014) pues resultan rentables y adecuadas para la eliminación de HC. Algunos de estos tratamientos son la inundación química y la recuperación mejorada de petróleo por microorganismos (MEOR, por sus siglas en inglés) (Roy, Dutta, Pal, Gupta, \& Sarkar, 2018).

Aunque el proceso de inundación química mejora la recuperación del petróleo, la disposición final del producto resulta difícil y los reactivos utilizados pueden contaminar aún más el medio ambiente (Cássia et al., 2017). La tecnología MEOR utiliza el crecimiento de comunidades microbianas nativas de los reservorios de petróleo para mejorar la recuperación de los sitios contaminados (Sakthipriya, Doble, \& Sangwai, 2015), suelos y cuerpos de agua. En los suelos contaminados con hidrocarburos, se suele encontrar un fenómeno de restricción del intercambio gaseoso con la atmosfera. Esto inicia una serie de procesos físico-químicos, como la evaporación y penetración, que dependiendo del tipo de contaminante, temperatura, humedad, textura, composición del suelo y de la cantidad vertida de HC, pueden ser más o menos lentos, ocasionando un mayor impacto y toxicidad (Benavides et al., 2006).

En este contexto, la técnica de biorremediación de suelos es una de las alternativas más prominentes. Es un proceso amigable con el medio ambiente que conserva la estructura del suelo, requiere poca energía e implica la degradación en sustancias menos nocivas o la destrucción completa del contaminante.

Dentro de la gran variedad de técnicas que reúne la biorremediación, una de las principales resistencias a la biodegradación de hidrocarburos en sitios contaminados es la biodisponibilidad de la sustancia. Por ello, diversos microorganismos presentes en la microflora de estos sitios poseen la capacidad de producir biosurfactantes (Chebbi et al., 2017) y, así, logran facilitar la pseudo solubilización del contaminante y aceleran la degradación del mismo (Peele, Venkateswarulu, \& Tammineedi, 2018).

El papel de los biosurfactantes es el de mejorar el proceso de atenuación natural e intensificar la biodegradación de HC (Hassan et al., 2017). Sin embargo, aunque tengan características atractivas y prometedoras en comparación con las contrapartes sintéticas como: poca o nula toxicidad, capacidad de renovación de los recursos, facilidad de producción, alta actividad y degradabilidad natural, entre otras; los altos costos de manufactura, principalmente el del sustrato para su producción, hacen que esta no sea rentable a escala industrial.

Existen parámetros importantes que deben considerarse cuando se diseña una estrategia o metodología para incrementar la degradación de hidrocarburos facilitada por biosurfactantes ya que éstos afectan la producción y eficacia de la técnica (Rougeux, Ye, Oudot, \& Chal, 2005). Entre dichos factores se encuentra la concentración y relación de nutrientes (Carbono, Nitrógeno y Fósforo), temperatura, velocidad de agitación, pH, oxígeno disuelto y salinidad. El estudio y la selección de los valores correctos para cada uno de ellos resulta de vital importancia para mejorar el rendimiento de los procesos y disminuir los costos de producción de los biosurfactantes. Para poder lograr este objetivo se utilizan métodos estadísticos de optimización que permiten encontrar los valores adecuados que minimizan costos y mejorar la productividad (Abalos, Maximo, Manresa, \& Bastida, 2002; Joice \& Parthasarathi, 2014; Ohadi et al., 2017). Entre estos la metodología de superficie de respuesta (RSM) es la más conocida y ampliamente utilizada. Esto es debido a que su objetivo es el de hallar las condiciones óptimas del proceso, a diferencia de los diseños experimentales simples que pretenden hallar las mejores condiciones entre las ensayadas.

RSM es un conjunto de técnicas matemáticas y estadísticas que permiten modelar y analizar situaciones en las que una variable (respuesta) se encuentra influenciada por otras (factores) (Myers, Montgomery, \& Anderson Cook, 2016). Consiste en llevar a cabo el diseño de un experimento que permita hallar valores de la respuesta para así determinar el modelo matemático que mejor se ajuste a los mismos y, luego, establecer cuáles son los valores de los factores que maximizan dicha respuesta.

En este trabajo, se utilizó RSM para establecer cuál es la temperatura y concentración de glicerol que maximizan la producción de biosurfactantes a partir de una cepa de Pseudomonas sp. aislada de un sitio crónicamente contaminado con hidrocarburos en la ciudad de Campana, provincia de Buenos Aires. La optimización estadística se llevó a cabo utilizando un diseño central compuesto (CCD) a través de una metodología de superficie de respuesta.

\section{Materiales y Métodos}

\section{1.-Microorganismo}

El microorganismo utilizado pertenece al género Pseudomonas sp. (identificada como Ag HC) aislada de agua crónicamente contaminada con hidrocarburos en una destilería de petróleo de la ciudad de Campana. De acuerdo 
con el análisis del ARN16s, y la comparación de los resultados con las bases de datos EZBioCloud y SeqMatch RDP, esta bacteria posee un 99,4\% de identidad respecto de Pseudomonas granadensis. Diversas bacterias de este género han sido identificadas en bibliografía como productoras de biosurfactantes. (Aparna et al. 2012, Yan et al. 2012, Ben Belgacem et al. 2015, Sharma et al. 2015).

2.- Medio de cultivo y Fuente de carbono

El medio de cultivo utilizado fue un medio mínimo salino (MSM) previamente descripto (Shen, Stehmeier, \& Voordouw, 1998). MSM es un medio ampliamente utilizado para estudios de degradación de hidrocarburos en microorganismos aislados de muestras de suelo y su composición es: 4g de $\mathrm{NaNO}_{3} ; 1,5 \mathrm{~g}$ de $\mathrm{KH}_{2} \mathrm{PO}_{4} ; 0$,5g de $\mathrm{Na}_{2} \mathrm{HPO}_{4} ; 0,0011 \mathrm{~g}$ de $\mathrm{FeSO}_{4} \cdot 7 \mathrm{H}_{2} \mathrm{O} ; 0,2 \mathrm{~g}$ de $\mathrm{MgSO}_{4} \cdot 7 \mathrm{H}_{2} \mathrm{O} ; 0,0132 \mathrm{~g}$ de $\mathrm{CaCl}_{2} \cdot 2 \mathrm{H}_{2} \mathrm{O}$ por litro de solución. El pH del medio se ajustó a 7,00 \pm 0,02. Todos los reactivos utilizados fueron de calidad grado analítico (Biopack). En los experimentos se utilizó glicerol grado analítico como fuente de carbono y energía.

Para realizar los pre-cultivos de activación para los ensayos, se utilizó el medio de cultivo de lisogenia (LB, medio Luria-Bertani).

3.- Estimación de la producción de biomasa

Se estimó la producción de biomasa del microorganismo a partir del peso seco al cabo de 24 horas (CDW): Se centrifugaron $16 \mathrm{ml}$ de cada muestra a $13500 \mathrm{rpm}$ a $19^{\circ} \mathrm{C}$ durante 15 minutos. El sobrenadante obtenido fue utilizado para la medición de la tensión superficial. El pellet fue resuspendido con agua destilada, trasvasado a un tubo eppendorf de $1.5 \mathrm{ml}$, previamente pesado, y se volvió a centrifugar. Se descartó el sobrenadante y el pellet fue colocado en una estufa de secado a $80^{\circ} \mathrm{C}$ durante $24 \mathrm{hs}$, para eliminar la humedad presente en la muestra.

4.- Estimación de la producción de biosurfactantes

4.1.- Medición directa de la tensión superficial (ST) del sobrenadante de cultivo: con un tensiómetro digital (Biolin Scientific Holding AB) por el método del anillo de Du Nouy (Walter, Syldatk, \& Hausmann, 2010; Youssef, Duncan, Nagle, Savage, Knapp, \& McInerney, 2004; Abouseoud, Maachi, Amrane, Boudergua, \& Nabi, 2008). Se utilizaron $16 \mathrm{ml}$ de sobrenadante de cultivo centrifugado a $13500 \mathrm{rpm}$ y $19^{\circ} \mathrm{C}$ por 15 minutos en una microcentrífuga (Labnet International INC.) para llevar a cabo la medición. Se realizaron 12 medidas sobre cada muestra y se calculó la media y el desvío estándar (STD) de los resultados. Se utilizó como control del ensayo el sobrenadante del cultivo control.

4.2.- Precipitación ácida en dos etapas: se utilizaron $18 \mathrm{ml}$ de muestra que se centrifugaron a $13500 \mathrm{rpm}$ y $18^{\circ} \mathrm{C}$ por $15 \mathrm{~min}$ para la separación de la biomasa. Luego, el sobrenadante fue acidificado con una solución 1M de $\mathrm{HCl}$ hasta un pH 2 y vuelto a centrifugar a $13500 \mathrm{rpm}$ y $4^{\circ} \mathrm{C}$ por $20 \mathrm{~min}$. El pellet obtenido fue resuspendido en agua destilada, trasvasado a un tubo de peso conocido, acidificado con $\mathrm{HCl}$ y centrifugado en las mismas condiciones. Luego, se retiró el sobrenadante y el precipitado obtenido se secó por $1 \mathrm{~h}$ a $80^{\circ} \mathrm{C}$.

5.- Optimización del medio de cultivo para la producción de biosurfactantes utilizando una metodología de superficie de respuesta

Se adoptó un diseño central compuesto (CCD) para determinar un modelo empírico del efecto y las interacciones de dos factores (variables) en la producción de biosurfactantes (respuesta). Las variables seleccionadas fueron temperatura y concentración de glicerol, utilizado como fuente de carbono y energía. Como respuesta se utilizó la concentración de biosurfactante obtenida el día de cosecha. En la Tabla 1 se muestran los rangos de los componentes. Estos rangos fueron determinados en base a la capacidad del equipamiento del laboratorio y a la bibliografía (Silva et al. 2010, Cardoso Fontes et al. 2010).

\begin{tabular}{cccc}
\hline Factor & Mínimo & Medio & Máximo \\
\hline Concentración de Sustrato $(\%)$ & 5 & 7,5 & 10 \\
\hline Temperatura $\left({ }^{\circ} \mathrm{C}\right)$ & 20 & 25 & 30 \\
\hline
\end{tabular}

Tabla 1: Rango experimental de las variables independientes para el diseño central compuesto utilizado para optimizar la producción de biosurfactantes.

Se utilizó el software estadístico Minitab 17® para el diseño de los experimentos, para el análisis de regresión de los datos experimentales y para graficar la relación entre las variables. Los valores óptimos fueron obtenidos resolviendo la ecuación de regresión y analizando el gráfico de superficie de respuesta. La información obtenida a 
partir del diseño central compuesto fue sometida a un análisis de varianza (ANOVA) para verificar los errores y la significancia de cada parámetro. El comportamiento del sistema puede ser explicado a partir de una ecuación de cuadrática de la forma:

$$
\hat{Y}=b_{0}+b_{1} \cdot X_{1}+b_{2} \cdot X_{2}+b_{11} \cdot X_{1}^{2}+b_{22} \cdot X_{2}^{2}+b_{12} \cdot X_{1} \cdot X_{2}
$$

donde $\hat{Y}$ es la respuesta, $\mathrm{X}_{1}$ y $\mathrm{X}_{2}$ las variables independientes codificadas, $\mathrm{b}_{0}$ el termino de compensación, $\mathrm{b}_{1}$ y $\mathrm{b}_{2}$ los efectos lineares, $b_{11}$ y $b_{22}$ los efectos cuadráticos y $b_{12}$ el término de interacción.

Con respecto a los ensayos experimentales, se llevaron a cabo en Erlenmeyers de $250 \mathrm{ml}$ por $96 \mathrm{~h}$. El procedimiento de cultivo fue el siguiente:

. Pre cultivo de activación: En Erlenmeyers de $250 \mathrm{ml}$ con $60 \mathrm{ml}$ de medio LB, en un agitador orbital a $25^{\circ} \mathrm{C}$ y 135 rpm por 3 días.

. Preparación del inóculo: Se diluyó una alícuota del pre cultivo de activación en agua destilada hasta un valor de DO correspondiente a $0,200 \pm 0,010$ a $600 \mathrm{~nm}$.

. Cultivo en Erlenmeyer: Se realizó en un volumen final de $80 \mathrm{ml}$ por cada Erlenmeyer. Se utilizó MSM suplementado con glicerol (a diferentes concentraciones de acuerdo con el experimento) y 3,6ml de inóculo. El cultivo se desarrolló por el término de 4 días a 135rpm y a la temperatura correspondiente. Se tomaron muestras al inicio y a las 96h de cultivo, y se les midió CDW y valor de la tensión superficial. A la muestra obtenida el día de cosecha se le estimó la concentración de biosurfactante a partir de una doble precipitación ácida. El control utilizado fue un Erlenmeyer con 145,5ml de MSM suplementado con 4,5ml de glicerol.

\section{Resultados y Conclusiones}

Se realizó un análisis de regresión múltiple utilizando una metodología de superficie de respuesta (RSM) para ajustar la función de respuesta a los datos experimentales y para evaluar la influencia de las dos variables seleccionadas. Las combinaciones de variables ensayadas y los correspondientes resultados se muestran en la Tabla 2. Allí se puede observar que la mejor condición de las ensayadas, teniendo en cuenta la producción de biosurfactantes, resulta la combinación de los valores medios de cada variable $\left(25^{\circ} \mathrm{C}\right.$ y $\left.7,5 \%\right)$.

\begin{tabular}{cccc}
\hline $\begin{array}{c}\text { Temperatura } \\
\left({ }^{\circ} \mathrm{C}\right)\end{array}$ & $\begin{array}{c}\text { Conc. de Sustrato } \\
(\% \mathrm{v} / \mathrm{v})\end{array}$ & $\begin{array}{c}\text { Conc. de Biosurfactante } \\
(\mathrm{g} / \mathrm{l})\end{array}$ & $\begin{array}{c}\text { Conc. de Biomasa } \\
(\mathrm{g} / \mathrm{l})\end{array}$ \\
\hline 17,9 & 7,50 & 0,10575 & 2,42813 \\
\hline 20,0 & 5,00 & 0,01556 & 0,15771 \\
\hline 20,0 & 10,00 & 0,04097 & 0,31146 \\
\hline 25,0 & 3,96 & 0,03821 & 0,72125 \\
\hline 25,0 & 7,50 & 0,42481 & 1,16333 \\
\hline 25,0 & 7,50 & 0,40234 & 1,10922 \\
\hline 25,0 & 7,50 & 0,44622 & 1,15998 \\
\hline 25,0 & 7,50 & 0,39987 & 1,10032 \\
\hline 25,0 & 7,50 & 0,41331 & 1,11000 \\
\hline 25,0 & 11,03 & 0,07467 & 0,62625 \\
\hline 30,0 & 5,00 & 0,11648 & 0,78771 \\
\hline 30,0 & 10,00 & 0,04115 & 0,55479 \\
\hline 32,1 & 7,50 & 0,23189 & 1,47000 \\
\hline
\end{tabular}

Tabla 2: Combinación de variables ensayadas para optimizar la producción de biosurfactantes a partir del cultivo de Ag HC. Se muestran también la concentración de biomasa alcanzada en cada caso.

La utilización de la RSM para estimar los parámetros óptimos condujo a una relación empírica entre la concentración de biosurfactante y las variables. El polinomio que mejor ajusta dichos datos es el siguiente:

$$
Y=-5,46+0,3053 . X_{1}+0,5283 . X_{2}-0,00566 \cdot X_{1}^{2}-0,03187 . X_{2}^{2}-0,00201 \cdot X_{1} \cdot X_{2}
$$

donde $\mathrm{Y}$ es la concentración de biosurfactante, $\mathrm{X}_{1}$ la temperatura y $\mathrm{X}_{2}$ la concentración de sustrato. 
La evaluación del modelo empírico fue realizada utilizando ANOVA (Figura 1). Con respecto al error, se puede observar que su valor es muy bajo $(0,009189)$ lo que indica que una muy buena reproducibilidad de los datos experimentales. Además, se puede observar que los términos cuadráticos del modelo son estadísticamente significativos $(\mathrm{p}<0,05)$, es decir, que afectan significativamente la respuesta. El término cuadrático de la concentración de sustrato utilizada resultó el más importante en este sentido.

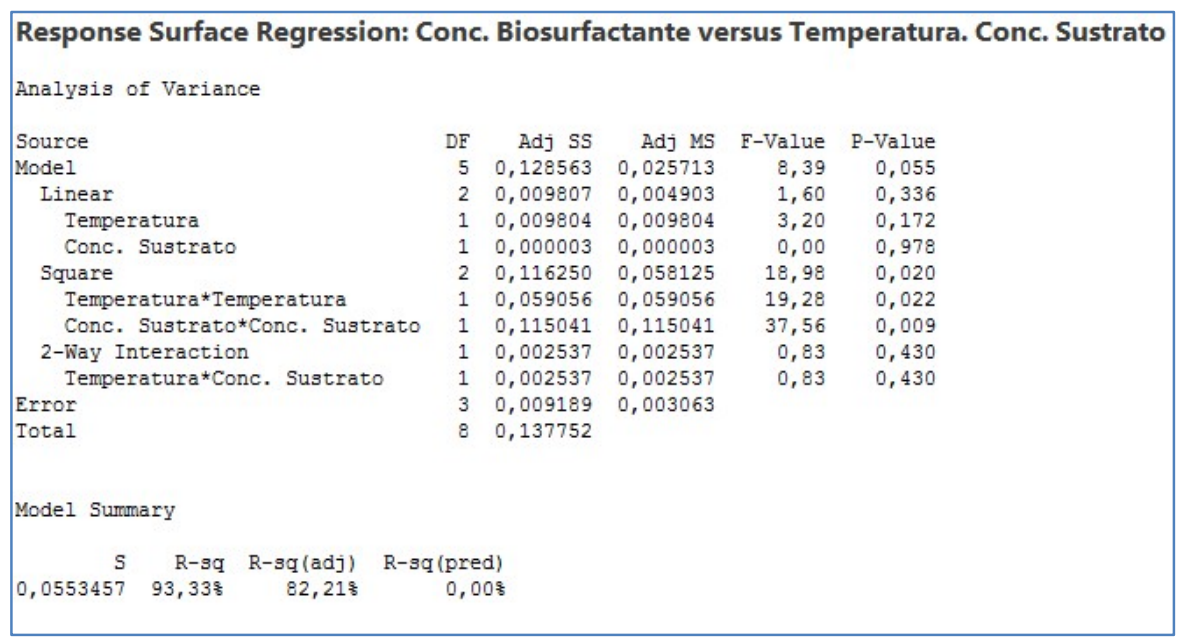

Figura 1: Análisis de la varianza para la concentración de biosurfactante

Sumado a esto, el valor del coeficiente de correlación, $\mathrm{R}^{2}=0.9333$, indica que menos del $7 \%$ de la variación total no puede ser explicada por el modelo empírico, por lo que el modelo es significativo y puede utilizarse para describir adecuadamente la producción de biosurfactantes.

En la Figura 2 se observa el gráfico de superficie y el de contorno para la producción de biosurfactantes, con temperatura y concentración de glicerol como factores. Allí puede observarse la interacción entre estas dos variables y cómo influyen en la respuesta. En la superficie de respuesta (Figura 2: izquierda) se observa que la máxima producción del biosurfactante se obtiene a medida que tanto la temperatura como la concentración de sustrato se acercan a sus valores medios, y que, si se aumentan o disminuyen estos valores, la producción del tensioactivo decae con cierta rapidez. Además, el gráfico de contorno (Figura 2: derecha) permite observar una fuerte interacción entre las variables, esto hace que no sea posible predecir el comportamiento de la producción del biosurfactante estudiando el cambio de una de las variables por vez, sino que deben evaluarse simultáneamente.

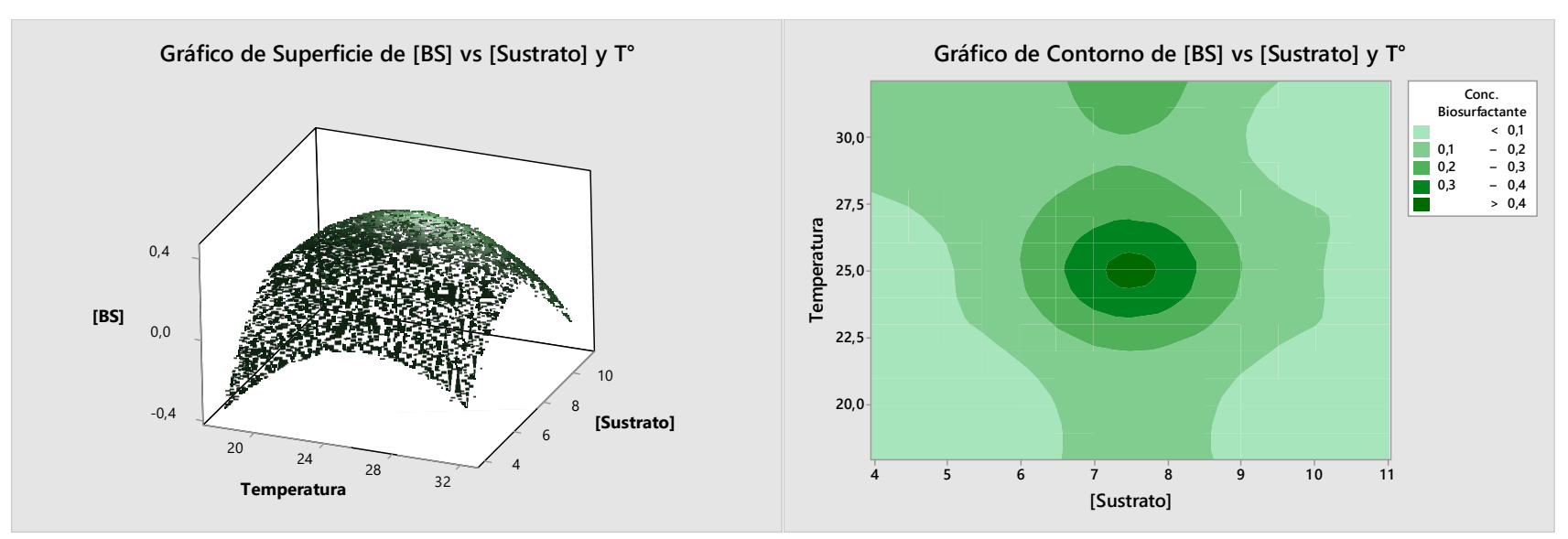

Figura 2: Superficie de respuesta (izquierda) y gráfico de contorno (derecha) de la concentración de biosurfactante en función de la temperatura y la concentración de glicerol.

En base a lo observado a partir del diseño experimental, la condición de $25,9^{\circ} \mathrm{C}$ y 7,6\% v/v de concentración de sustrato resulta la mejor opción para lograr la maximización de la producción de biosurfactantes. 
En conclusión, se ha aplicado un método de diseño factorial en el que se analizó la influencia de la temperatura y la concentración de glicerol en la producción de biosurfactantes en el cultivo de una cepa de Pseudomonas sp. aislada de un sitio crónicamente contaminado, así como la interacción de estos factores. Se obtuvo un polinomio de ajuste de los datos experimentales que modela más del $93 \%$ de la variación total obtenida a partir de los mismos. Este puede utilizarse para predecir cómo evolucionará el proceso de producción al modificar alguna de las variables analizadas. Además, a partir del modelo, se ha determinado las condiciones de temperatura $\left(25,9^{\circ} \mathrm{C}\right)$ y concentración de glicerol $(7,6 \% \mathrm{v} / \mathrm{v})$ que maximizan la producción de biosurfactante $(0,43 \mathrm{~g} / \mathrm{l})$.

\section{Referencias}

Abalos, A., Maximo, F., Manresa, M. A., \& Bastida, J. (2002). Utilization of response surface methodology to optimize the culture media for the production of rhamnolipids by Pseudomonas aeruginosa AT10. Journal of Chemical Technology and Biotechnology, 77(7), 777-784. https://doi.org/10.1002/jctb.637

Adams, G. O., Fufeyin, P. T., Okoro, S. E., Ehinomen, I., \& Biology, E. (2015). Bioremediation , Biostimulation and Bioaugmention: A Review. Journal of Environmental Bioremediation and Biodegradation, 3(1), $28-39$. https://doi.org/10.12691/ijebb-3-1-5

Allard, A., \& Neilson, A. H. (1997). Bioremediation of Organic Waste Sites : A Critical Review of Microbiological Aspects. 39(4), 253-285.

Benavides, J., Mesa, L. De, Quintero, G., Liliana, A., Vizcaíno, G., \& Jaimes, D. C. (2006). Bioremediación de suelos contaminados con hidrocarburos derivados del petróleo. 4(5), 82-90.

Bioremediation_A review of applications and problems to be solved.pdf. (n.d.).

Cássia, R. De, Soares, F., Almeida, D. G., Meira, H. M., Silva, E. J., Farias, C. B. B., ... Sarubbo, L. A. (2017). Biocatalysis and Agricultural Biotechnology Production and characterization of a new biosurfactant from Pseudomonas cepacia grown in low-cost fermentative medium and its application in the oil industry. 12(December 2016), 206-215. https://doi.org/10.1016/j.bcab.2017.09.004

Chebbi, A., Hentati, D., Zaghden, H., Baccar, N., Rezgui, F., Chalbi, M., ... Chamkha, M. (2017). International Biodeterioration \& Biodegradation Polycyclic aromatic hydrocarbon degradation and biosurfactant production by a newly isolated Pseudomonas sp . strain from used motor oil-contaminated soil. International Biodeterioration \& Biodegradation, 122, 128-140. https://doi.org/10.1016/j.ibiod.2017.05.006

Doheim, M. M. (2014). Selection of Pseudomonas aeruginosa for biosurfactant production and studies of its antimicrobial activity. Egyptian Journal of Petroleum, 23(1), 1-6. https://doi.org/10.1016/j.ejpe.2014.02.001

Hassan, A., Khan, A., Tanveer, S., Alia, S., Anees, M., Sultan, A., ... Yousaf, S. (2017). Role of nutrients in bacterial biosurfactant production and effect of biosurfactant production on petroleum hydrocarbon biodegradation. Ecological Engineering, 104, 158-164. https://doi.org/10.1016/j.ecoleng.2017.04.023

Joice, P. A., \& Parthasarathi, R. (2014). Optimization of biosurfactant production from Pseudomonas aeruginosa PBSC1. International Journal of Current Microbiology and Applied Sciences, 3(9), 140-151.

Myers, R., Montgomery, D., \& Anderson Cook, C. (2016). Response Surface Methodology: Process and product optimization using designed experiments (4th Ed.). Wiley and Sons.

Ohadi, M., Dehghannoudeh, G., Shakibaie, M., Banat, I. M., Pournamdari, M., \& Forootanfar, H. (2017). Isolation, characterization, and optimization of biosurfactant production by an oil-degrading Acinetobacter junii B6 isolated from an Iranian oil excavation site. Biocatalysis and Agricultural Biotechnology, 12, 1-9. https://doi.org/10.1016/j.bcab.2017.08.007

Peele, A., Venkateswarulu, T. C., \& Tammineedi, J. (2018). Role of biosurfactants in bioremediation of oil pollutiona review. Petroleum, 4(3), 241-249. https://doi.org/10.1016/j.petlm.2018.03.007

Rougeux, G., Ye, C., Oudot, J., \& Char, C. H. (2005). Effects of nutrient concentration on the biodegradation of crude oil and associated microbial populations in the soil. 37, 1490-1497. https://doi.org/10.1016/j.soilbio.2005.01.012

Roy, A., Dutta, A., Pal, S., Gupta, A., \& Sarkar, J. (2018). Bioresource Technology Biostimulation and bioaugmentation of native microbial community accelerated bioremediation of oil re fi nery sludge. 253(November 2017), 22-32. https://doi.org/10.1016/j.biortech.2018.01.004

Sakthipriya, N., Doble, M., \& Sangwai, J. S. (2015). Journal of Industrial and Engineering Chemistry Biosurfactant from Pseudomonas species with waxes as carbon source - Their production , modeling and properties. Journal of Industrial and Engineering Chemistry, 31,100-111. https://doi.org/10.1016/j.jiec.2015.06.013 